MATHEMATICS OF COMPUTATION

Volume 67, Number 224, October 1998, Pages 1565-1575

S 0025-5718(98)00987-9

\title{
THE TODA MOLECULE EQUATION AND THE $\varepsilon$-ALGORITHM
}

\author{
ATSUSHI NAGAI, TETSUJI TOKIHIRO, AND JUNKICHI SATSUMA
}

\begin{abstract}
One of the well-known convergence acceleration methods, the $\varepsilon$-algorithm is investigated from the viewpoint of the Toda molecule equation. It is shown that the error caused by the algorithm is evaluated by means of solutions for the equation. The acceleration algorithm based on the discrete

Toda molecule equation is also presented.
\end{abstract}

Discrete integrable systems play important roles in the field of numerical analysis. Matrix eigenvalue algorithms ([11], [19], [20]) and convergence acceleration methods ([1], [14], [15]) are typical examples. We here focus our attention mainly on the convergence acceleration methods and investigate both qualitatively and quantitatively their features from the viewpoint of discrete integrable systems. In particular, we show that there is a strong relation between the $\varepsilon$-algorithm and the discrete Toda molecule equation.

Let us first consider the equation of motion given by

$$
\begin{aligned}
\frac{\mathrm{d}^{2} Q_{1}}{\mathrm{~d} t^{2}} & =-\mathrm{e}^{-\left(Q_{2}-Q_{1}\right)}, \\
\frac{\mathrm{d}^{2} Q_{n}}{\mathrm{~d} t^{2}} & =-\mathrm{e}^{-\left(Q_{n+1}-Q_{n}\right)}+\mathrm{e}^{-\left(Q_{n}-Q_{n-1}\right)} \quad(n=1,2, \ldots, N-1), \\
\frac{\mathrm{d}^{2} Q_{N}}{\mathrm{~d} t^{2}} & =\mathrm{e}^{-\left(Q_{N}-Q_{N-1}\right)} .
\end{aligned}
$$

Equation (1) is obtained by imposing the formal boundary condition

$$
Q_{0}=-\infty, Q_{N+1}=\infty
$$

in the Toda lattice equation [21] and is also called the Toda molecule equation. Owing to its boundary condition (2), each particle moves freely and the distance between two neighboring particles becomes infinite as $t \rightarrow \infty$. Under Flaschka's change of variables [7],

$$
a_{n}=\frac{1}{2} \exp \left(-\frac{Q_{n+1}-Q_{n}}{2}\right), b_{n}=\frac{1}{2} \dot{Q}_{n}=\frac{1}{2} \frac{\mathrm{d} Q}{\mathrm{~d} t},
$$

equation (1) is rewritten in the following matrix differential equation,

$$
\frac{\mathrm{d} X}{\mathrm{~d} t}=[X, B]
$$

Received by the editor May 20, 1996 and, in revised form, November 5, 1996 and February 13, 1997.

1991 Mathematics Subject Classification. Primary 58F07, 65B10.

Key words and phrases. Toda molecule equation, $\varepsilon$-algorithm, Padé approximation, continued fraction.

(C)1998 American Mathematical Society 
where

$$
\begin{aligned}
X(t)= & {\left[\begin{array}{ccccc}
b_{1} & a_{1} & & & 0 \\
a_{1} & b_{2} & a_{2} & & \\
& a_{2} & \ddots & \ddots & \\
& & \ddots & b_{N-1} & a_{N-1} \\
\mathbf{0} & & & a_{N-1} & b_{N}
\end{array}\right], } \\
B(t)= & {\left[\begin{array}{ccccc}
0 & a_{1} & & & \mathbf{0} \\
-a_{1} & 0 & a_{2} & & \\
& -a_{2} & \ddots & \ddots & \\
& & \ddots & 0 & a_{N-1} \\
\mathbf{0} & & & -a_{N-1} & 0
\end{array}\right] . }
\end{aligned}
$$

In 1982, Symes [20] pointed out that the time evolution of the Toda molecule equation is equivalent to the iteration of the $\mathrm{QR}$ algorithm. In detail, if the matrix $\mathrm{e}^{-X(t)}$ has the QR decomposition

$$
\mathrm{e}^{-X(t)}=Q(t) R(t)
$$

then the relation

$$
R(t) Q(t)=\mathrm{e}^{-X(t+1)}
$$

holds. This equivalence is also interpreted as follows. Since each particle moves freely, the quantities $a_{n}(t)$ tend to zero as $t \rightarrow \infty$, which is nothing but diagonalization of the matrix $X(t)$ in the $\mathrm{QR}$ algorithm. This may give one reason for which the Toda molecule system and the QR algorithm coincide even though they have different backgrounds.

Can we give a similar interpretation with respect to convergence acceleration methods, which have been pointed out to relate with discrete integrable systems? The purpose of this paper is to establish a relation between the $\varepsilon$-algorithm and the Toda molecule equation, to give a physical reason for convergence acceleration from the equation, and to estimate quantitatively the error caused by the algorithm by means of the solution for the equation.

The $\varepsilon$-algorithm

$$
\varepsilon_{0}^{(m)}=0, \varepsilon_{1}^{(m)}=S_{m},\left(\varepsilon_{n+1}^{(m)}-\varepsilon_{n-1}^{(m+1)}\right)\left(\varepsilon_{n}^{(m+1)}-\varepsilon_{n}^{(m)}\right)=1
$$

was first proposed by Wynn [25] as a convergence accelerator for a given sequence $\left\{S_{m}\right\}$ and is one of the most powerful acceleration schemes. It should be noted that there had already been pioneering works by Wynn ([26], [27], [28], [29], [30]) before soliton equations were actively studied. He derived many semi-discrete soliton equations by considering infinitesimal analogues of lorenze algorithms, which he called confluent forms, and described their interesting properties which may be deduced from those of the algorithms themselves. One of them, known as a confluent form of the $\varepsilon$-algorithm, is given by

$$
\left(\varepsilon_{n+1}(t)-\varepsilon_{n-1}(t)\right) \dot{\varepsilon}_{n}(t)=1 .
$$

In terms of soliton theory, equation (10) reduces to the Bäcklund transformation of the Toda molecule equation (1),

$$
\dot{N}_{n}(t)=N_{n}(t)\left(N_{n-1}(t)-N_{n+1}(t)\right)
$$


through dependent variable transformation,

$$
N_{n}(t)=\dot{\varepsilon}_{n}(t) \dot{\varepsilon}_{n+1}(t) .
$$

Equation (11) is also called the Lotka-Volterra equation ([9], [10]). The $\varepsilon$-algorithm itself is considered as an integrable discretization of the KdV equation by taking an appropriate continuous limit of both discrete variables $m$ and $n$ ([15], [14]).

Since the $\varepsilon$-algorithm (9) is regarded as a full discrete equation, here we consider the time discrete version of the Toda molecule equation [11],

$$
\left\{\begin{aligned}
I_{n+1}^{(m)} V_{n}^{(m)} & =I_{n}^{(m+1)} V_{n}^{(m+1)} \\
I_{n}^{(m+1)}-I_{n}^{(m)} & =V_{n}^{(m)}-V_{n-1}^{(m+1)}
\end{aligned}\right.
$$

It should also be noted, as was pointed out by Watkins and Elsner [24], that Rutishauser [17] had already derived the Toda molecule equation (1) before Toda by considering an infinitesimal analogue of equation (13). Equation (13) is well known as the QD algorithm or the LR algorithm in the field of numerical analysis. It also finds many more applications in the field of engineering and mathematics ([12], [13]). The quantities $I_{n}^{(m)}$ and $V_{n}^{(m)}$ correspond to $\dot{Q}_{n}(t)$ and $\exp \left(-\left(Q_{n+1}(t)-Q_{n}(t)\right)\right)$, respectively, and the variable $m$ stands for the time variable. They are given by ([10], [11])

$$
\begin{array}{r}
I_{n}^{(m)}=\frac{\tau_{n-1}^{(m)} \tau_{n}^{(m+1)}}{\tau_{n}^{(m)} \tau_{n-1}^{(m+1)}}, V_{n}^{(m)}=\frac{\tau_{n+1}^{(m)} \tau_{n-1}^{(m+1)}}{\tau_{n}^{(m)} \tau_{n}^{(m+1)}}, \\
\tau_{n}^{(m)}=\sum_{1 \leq i_{1}<\cdots<i_{n} \leq N} V\left(P_{i_{1}}, \cdots, P_{i_{n}}\right)^{2} C_{i_{1}} P_{i_{1}}^{m} \cdots C_{i_{n}} P_{i_{n}}^{m}, \\
V\left(P_{i_{1}}, P_{i_{2}}, \cdots, P_{i_{k}}\right)=\left|\begin{array}{cccc}
1 & 1 & \cdots & 1 \\
P_{i_{1}} & P_{i_{2}} & \cdots & P_{i_{k}} \\
\vdots & \vdots & & \vdots \\
P_{i_{1}}^{k-1} & P_{i_{2}}^{k-1} & \cdots & P_{i_{k}}^{k-1}
\end{array}\right|,
\end{array}
$$

where $P_{1}, P_{2}, \cdots, P_{N}$ and $\left(C_{1}: C_{2}: \cdots: C_{N}\right) \in \mathrm{P}^{N-1}$ are $2 N-1$ independent parameters determined by independent initial data ${ }^{1} V_{1}^{(0)}, \cdots, V_{N-1}^{(0)}, I_{1}^{(0)}, \cdots, I_{N}^{(0)}$. The parameters $P_{1}, P_{2}, \cdots, P_{N}$ are eigenvalues of the $N \times N$ matrix,

$$
X^{(0)}=\left[\begin{array}{ccccc}
I_{1}^{(0)} & 1 & & & 0 \\
I_{1}^{(0)} V_{1}^{(0)} & I_{2}^{(0)}+V_{1}^{(0)} & 1 & & \\
& I_{2}^{(0)} V_{2}^{(0)} & \ddots & \ddots & \\
0 & & \ddots & \ddots & 1 \\
0 & & & I_{N-1}^{(0)} V_{N-1}^{(0)} & I_{N}^{(0)}+V_{N-1}^{(0)}
\end{array}\right],
$$

and satisfy, without loss of generality, the inequalities,

$$
\left|P_{1}\right|>\left|P_{2}\right|>\cdots>\left|P_{N}\right|
$$

\footnotetext{
${ }^{1}$ Two sets of parameters $\left(P_{1}, \cdots, P_{N}, C_{1}: C_{2}: \cdots: C_{N}\right)$ and $\left(V_{1}^{(0)}, \cdots, V_{N-1}^{(0)}, I_{1}^{(0)}, \cdots, I_{N}^{(0)}\right)$ are related by a simultaneous system of $2 N-1$ independent algebraic equations.
} 
when $I_{j}^{(0)} V_{j}^{(0)} \neq 0$ holds for any $j=1,2, \cdots, N-1$. We see from equation (14) that

$$
I_{n}^{(m)}=P_{n}+O\left(\max \left[\frac{P_{n}}{P_{n-1}}, \frac{P_{n+1}}{P_{n}}\right]^{m}\right) \rightarrow P_{n}
$$

$$
V_{n}^{(m)}=\frac{C_{n+1} \prod_{i=1}^{n}\left(P_{n+1}-P_{i}\right)^{2}}{C_{n} P_{n} \prod_{i=1}^{n-1}\left(P_{n}-P_{i}\right)^{2}}\left(\frac{P_{n+1}}{P_{n}}\right)^{m}+O\left(\max \left[\frac{P_{n+1}}{P_{n-1}}, \frac{P_{n+2}}{P_{n}}\right]^{m}\right) \rightarrow 0,
$$

as $m \rightarrow \infty$. Equation (20) shows that each quantity $V_{n}^{(m)}$ converges exponentially, or in the order of $O\left(\left(P_{n+1} / P_{n}\right)^{m}\right)$, to zero as $m \rightarrow \infty$.

The establishment of a relation between the $\varepsilon$-algorithm and the discrete Toda molecule equation consists of two parts: first, the $\varepsilon$-algorithm and the Padé approximation and second, the discrete Toda molecule equation and continued fractions.

First of all, we consider the Padé approximation and its relation to the $\varepsilon$-algorithm. Let

$$
f(x)=\sum_{i=0}^{\infty} c_{i} x^{i}
$$

be a given formal power series such that an infinite series $f(1)=\sum_{i=0}^{\infty} c_{i}$ is a convergent, necessary condition which is given by

$$
\varlimsup_{m \rightarrow \infty}\left(\left|c_{m}\right|\right)^{1 / m} \leq 1
$$

Padé approximation of $f(x)$ consists in finding a rational function $R^{[m / n]}(x)$ with the numerator of the $m$-th degree and the denominator of the $n$-th degree,

$$
R^{[m / n]}(x)=\frac{a_{0}+a_{1} x+a_{2} x^{2}+\cdots+a_{m} x^{m}}{b_{0}+b_{1} x+b_{2} x^{2}+\cdots+b_{n} x^{n}} \equiv \frac{P^{[m / n]}(x)}{Q^{[m / n]}(x)},
$$

such that

$$
Q^{[m / n]}(x) f(x)-P^{[m / n]}(x)=O\left(x^{m+n+1}\right)
$$

holds. Putting $b_{0}=1$, we see that equation (24) is equivalent to the following Padé equations for $\left(a_{0}, a_{1}, \ldots, a_{m}\right)$ and $\left(b_{1}, b_{2}, \ldots, b_{n}\right)$ :

$$
\left(\begin{array}{cccc}
c_{m-n+1} & c_{m-n+2} & \cdots & c_{m} \\
c_{m-n+2} & c_{m-n+3} & \cdots & c_{m+1} \\
c_{m-n+3} & c_{m-n+4} & \cdots & c_{m+2} \\
\vdots & \vdots & & \vdots \\
c_{m} & c_{m+1} & \cdots & c_{m+n-1}
\end{array}\right)\left(\begin{array}{c}
b_{n} \\
b_{n-1} \\
b_{n-2} \\
\vdots \\
b_{1}
\end{array}\right)=-\left(\begin{array}{c}
c_{m+1} \\
c_{m+2} \\
c_{m+3} \\
\vdots \\
c_{m+n}
\end{array}\right)
$$




$$
\begin{aligned}
& a_{0}=c_{0}, \\
& a_{1}=c_{1}+b_{1} c_{0}, \\
& a_{2}=c_{2}+b_{1} c_{1}+b_{2} c_{0} \text {, } \\
& a_{m}=c_{m}+\sum_{i=1}^{\min (m, n)} b_{i} c_{m-i}
\end{aligned}
$$

Solving linear equations (25) and (26), we have a Padé approximant $R^{[m / n]}(x)$ for $f(x)$. We see from Cramer's formula that the numerator and the denominator of the Padé approximant $R^{[m / n]}(x)$ are expressed as the following determinant form:

$$
\begin{aligned}
& P^{[m / n]}(x)=\left|\begin{array}{cccc}
c_{m-n+1} & c_{m-n+2} & \cdots & c_{m+1} \\
c_{m-n+2} & c_{m-n+3} & \cdots & c_{m+2} \\
\vdots & \vdots & & \vdots \\
c_{m} & c_{m+1} & \cdots & c_{m+n} \\
\sum_{i=0}^{m-n} c_{i} x^{n+i} & \sum_{i=0}^{m-n+1} c_{i} x^{n+i-1} & \cdots & \sum_{i=0}^{m} c_{i} x^{i}
\end{array}\right| \text {, } \\
& Q^{[m / n]}(x)=\left|\begin{array}{cccc}
c_{m-n+1} & c_{m-n+2} & \cdots & c_{m+1} \\
c_{m-n+2} & c_{m-n+3} & \cdots & c_{m+2} \\
\vdots & \vdots & & \vdots \\
c_{m} & c_{m+1} & \cdots & c_{m+n} \\
x^{n} & x^{n-1} & \cdots & 1
\end{array}\right|
\end{aligned}
$$

Substitution of $x=1$ in equations (27) and (28) gives

$$
R^{[m / n]}(1)=\frac{P^{[m / n]}(1)}{Q^{[m / n]}(1)}=\frac{\left|\begin{array}{cccc}
S_{m-n} & S_{m-n+1} & \cdots & S_{m} \\
S_{m-n+1} & S_{m-n+2} & \cdots & S_{m+1} \\
\vdots & \vdots & & \vdots \\
S_{m} & S_{m+1} & \cdots & S_{m+n}
\end{array}\right|}{\left|\begin{array}{cccc}
\Delta^{2} S_{m-n} & \Delta^{2} S_{m-n+1} & \cdots & \Delta^{2} S_{m-1} \\
\Delta^{2} S_{m-n+1} & \Delta^{2} S_{m-n+2} & \cdots & \Delta^{2} S_{m} \\
\vdots & \vdots & & \vdots \\
\Delta^{2} S_{m-1} & \Delta^{2} S_{m} & \cdots & \Delta^{2} S_{m+n-2}
\end{array}\right|},
$$

where $S_{m}$ is a summation of $c_{i}$ 's,

$$
S_{m}=\sum_{i=0}^{m} c_{i}
$$

and $\Delta$ is the forward difference operator given by $\Delta a_{k}=a_{k+1}-a_{k}$. Equation (29) immediately reminds us of the Shanks transformation [18]. In fact, the Padé approximation and the $\varepsilon$-algorithm are related by $([2],[8])$

$$
\varepsilon_{2 n+1}^{(m)}=R^{[m+n / n]}(1)
$$


Next we discuss the relation between the Padé approximation and the discrete Toda molecule equation via continued fractions. ${ }^{2}$ Let us begin with the following theorem.

Theorem 1 (Perron [16, pp. 447-459]). Let

$$
\begin{aligned}
T_{m}(x)= & \left\{R^{[m / 0]}(x), R^{[m+1 / 0]}(x), R^{[m+1 / 1]}(x), R^{[m+2 / 1]}(x),\right. \\
& \left.R^{[m+2 / 2]}(x), \ldots, R^{[m+n / n]}(x), R^{[m+n+1 / n]}(x), \ldots\right\} \quad(m \geq 0)
\end{aligned}
$$

denote a sequence of Padé approximants for $f(x)=\sum_{i=0}^{\infty} c_{i} x^{i}$, any two consecutive elements of which are different. Then there exists a continued fraction,

$$
c_{0}+c_{1} x+\cdots+c_{m} x^{m}+\frac{c_{m+1} x^{m+1} \mid}{\mid 1}+\frac{d_{2} x \mid}{\mid 1}+\frac{d_{3} x \mid}{\mid 1}+\cdots
$$

such that the $n$-th approximant of equation (33) is equal to the $(n+1)$-st term of $T_{m}(x)$ for any $n \geq 1$.

Let $g_{m}(x)$ be a continued fraction whose $n$-th approximant is equal to the $(n+1)$-st term of $T_{m}(x)$ for any $n$. We write $g_{m}(x)$ as follows:

$$
\begin{aligned}
g_{m}(x)= & c_{0}+c_{1} x+\cdots+c_{m} x^{m}+\frac{c_{m+1} x^{m+1} \mid}{\mid 1}-\frac{I_{1}^{(m+1)} x \mid}{\mid 1} \\
& -\frac{V_{1}^{(m+1)} x \mid}{\mid 1}-\frac{I_{2}^{(m+1)} x \mid}{\mid 1}-\frac{V_{2}^{(m+1)} x \mid}{\mid}-\frac{I_{3}^{(m+1)} x \mid}{\mid 1}-\cdots
\end{aligned}
$$

Taking the even part of equation (34), we have another continued fractional representation for $g_{m}(x)$,

$$
\begin{aligned}
g_{m}(x)=c_{0} & +c_{1} x+\cdots c_{m} x^{m}+\frac{c_{m+1} x^{m+1} \mid}{\mid 1-I_{1}^{(m+1)} x} \\
& +\frac{-I_{1}^{(m+1)} V_{1}^{(m+1)} x^{2} \mid}{\mid 1-\left(I_{2}^{(m+1)}+V_{1}^{(m+1)}\right) x}+\frac{-I_{2}^{(m+1)} V_{2}^{(m+1)} x^{2} \mid}{\mid 1-\left(I_{3}^{(m+1)}+V_{2}^{(m+1)}\right) x}+\cdots
\end{aligned}
$$

In the same way, we may consider a continued fraction,

$$
\begin{aligned}
g_{m-1}(x)= & c_{0}+c_{1} x+\cdots+c_{m-1} x^{m-1}+\frac{c_{m} x^{m} \mid}{\mid 1}-\frac{I_{1}^{(m)} x \mid}{\mid 1} \\
& -\frac{V_{1}^{(m)} x \mid}{\mid 1}-\frac{I_{2}^{(m)} x \mid}{\mid 1}-\frac{V_{2}^{(m)} x \mid}{\mid 1}-\frac{I_{3}^{(m)} x \mid}{\mid 1}-\cdots,
\end{aligned}
$$

\footnotetext{
${ }^{2}$ Elementary properties of continued fractions are given, for example, in [16] and [23].
} 
the $n$-th approximant of which equals the $(n+1)$-st term of $T_{m-1}(x)$. Taking the odd part of equation (36), we have

$$
\begin{aligned}
g_{m-1}(x)= & c_{0}+c_{1} x+\cdots c_{m} x^{m}+\frac{c_{m} I_{1}^{(m)} x^{m+1} \mid}{\mid 1-\left(I_{1}^{(m)}+V_{1}^{(m)}\right) x} \\
& +\frac{-I_{2}^{(m)} V_{1}^{(m)} x^{2} \mid}{\mid 1-\left(I_{2}^{(m)}+V_{2}^{(m)}\right) x}+\frac{-I_{3}^{(m)} V_{2}^{(m)} x^{2} \mid}{\mid 1-\left(I_{3}^{(m)}+V_{3}^{(m)}\right) x}+\cdots
\end{aligned}
$$

The $n$-th approximants of equations (35) and (37) are equal to $(2 n+1)$-st term of $T_{m}(x)$ and $(2 n+2)$-nd term of $T_{m-1}(x)$, respectively, both of which is nothing but $R^{[m+n / n]}(x)$. Therefore, two continued fractions (35) and (37) are equivalent. This leads to the following relation between the quantities $\left\{I_{n}^{(m)}\right\}$ and $\left\{V_{n}^{(m)}\right\}$,

$$
\left\{\begin{aligned}
I_{n+1}^{(m)} V_{n}^{(m)}= & I_{n}^{(m+1)} V_{n}^{(m+1)}, \\
I_{n}^{(m)}+V_{n}^{(m)}= & I_{n}^{(m+1)}+V_{n-1}^{(m+1)}, \\
V_{0}^{(m)}=0, & I_{1}^{(m)}=c_{m+1} / c_{m},
\end{aligned}\right.
$$

which is nothing but the discrete Toda molecule equation, or equivalently the QD or LR algorithm. It should be noted that the solutions (14) are recovered by putting

$$
c_{m}=\sum_{i=1}^{N} C_{i} P_{i}^{m}
$$

in equation (38). In particular, the quantities $I_{n}^{(0)}$ and $V_{n}^{(0)}$ are related to an initial sequence $\left\{c_{m}\right\}$ by

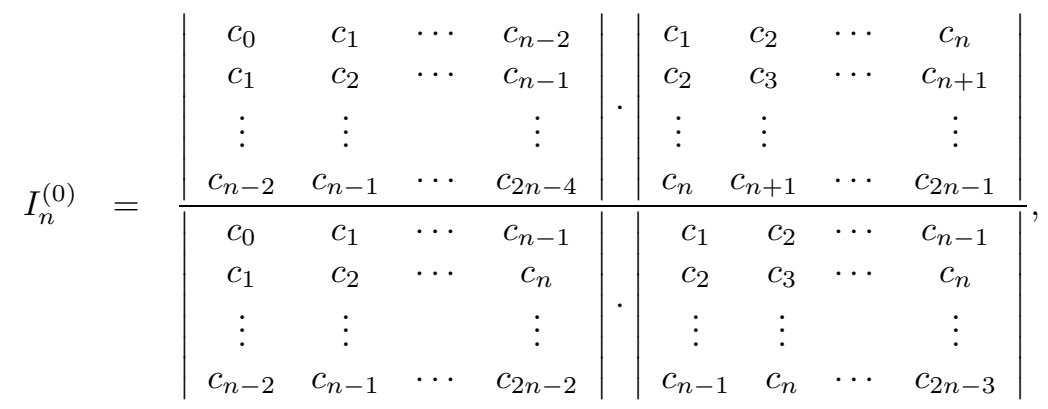

$$
\begin{aligned}
& V_{n}^{(0)}=\frac{\left|\begin{array}{cccc}
c_{0} & c_{1} & \cdots & c_{n} \\
c_{1} & c_{2} & \cdots & c_{n+1} \\
\vdots & \vdots & & \vdots \\
c_{n} & c_{n+1} & \cdots & c_{2 n}
\end{array}\right| \cdot\left|\begin{array}{cccc}
c_{1} & c_{2} & \cdots & c_{n-1} \\
c_{2} & c_{3} & \cdots & c_{n} \\
\vdots & \vdots & & \vdots \\
c_{n-1} & c_{n} & \cdots & c_{2 n-3}
\end{array}\right|}{\left|\begin{array}{cccc}
c_{0} & c_{1} & \cdots & c_{n-1} \\
c_{1} & c_{2} & \cdots & c_{n} \\
\vdots & \vdots & & \vdots \\
c_{n-1} & c_{n} & \cdots & c_{2 n-2}
\end{array}\right| \cdot\left|\begin{array}{cccc}
c_{1} & c_{2} & \cdots & c_{n} \\
c_{2} & c_{3} & \cdots & c_{n+1} \\
\vdots & \vdots & & \vdots \\
c_{n} & c_{n+1} & \cdots & c_{2 n-1}
\end{array}\right|}
\end{aligned}
$$


We see from the above discussion that the formal power series $f(x)=c_{0}+c_{1} x+$ $c_{2} x^{2}+c_{3} x^{3}+\cdots$ has a continued fractional representation,

$$
\begin{array}{r}
c_{0}+c_{1} x+\cdots+c_{m} x^{m}+\frac{c_{m+1} x^{m+1} \mid}{\mid 1}-\frac{I_{1}^{(m+1)} x \mid}{\mid}-\frac{V_{1}^{(m+1)} x \mid}{1} \\
-\frac{I_{2}^{(m+1)} x \mid}{\mid}-\frac{V_{2}^{(m+1)} x \mid}{\mid}-\frac{I_{3}^{(m+1)} x \mid}{\mid}-\frac{V_{3}^{(m+1)} x \mid}{\mid}-\cdots,
\end{array}
$$

and that each coefficient is governed by the discrete Toda molecule equation. Putting $x=1$ in equation (42), we have a continued fractional representation for an infinite series,

$$
\begin{aligned}
\sum_{i=0}^{\infty} c_{i}= & c_{0}+c_{1}+\cdots+c_{m}+\frac{c_{m+1} \mid}{\mid}-\frac{I_{1}^{(m+1)} \mid}{\mid}-\frac{V_{1}^{(m+1)} \mid}{1} \\
& -\frac{I_{2}^{(m+1)} \mid}{\mid}-\frac{V_{2}^{(m+1)} \mid}{\mid 1}-\frac{I_{3}^{(m+1)} \mid}{\mid}-\frac{V_{3}^{(m+1)} \mid}{\mid}-\cdots
\end{aligned}
$$

We again consider the discrete Toda molecule equation (13), in which the quantities $V_{n}^{(m)}$ decay exponentially to zero as $m \rightarrow \infty$. If we approximate $V_{n}^{(m+1)}=0$ in equation (43) for a certain $n \geq 1$, we have one approximation for the infinite series $\sum_{i=0}^{\infty} c_{i}$. The approximation of $V_{n}^{(m+1)}=0$ is equivalent to taking the $2 n$-th convergent of the continued fraction (43). From Theorem 1 , the $2 n$-th convergent of equation (43) equals $(2 n+1)$-st term of the sequence $T_{m}(1)$, namely $R^{[m+n / n]}(1)$.

Combining the above result with equation (31), we arrive at the following relation $^{3}$ between the $\varepsilon$-algorithm (9) and the discrete Toda molecule equation (13):

$$
\begin{aligned}
\varepsilon_{2 n+1}^{(m)}= & S_{m}+\frac{c_{m+1} \mid}{\mid 1}-\frac{I_{1}^{(m+1)} \mid}{\mid 1}-\frac{V_{1}^{(m+1)} \mid}{1}-\frac{I_{2}^{(m+1)} \mid}{1} \\
& -\frac{V_{2}^{(m+1)} \mid}{\mid}-\frac{I_{3}^{(m+1)} \mid}{\mid 1}-\frac{V_{3}^{(m+1)} \mid}{\mid 1}-\cdots-\frac{I_{n}^{(m+1)} \mid}{\mid} .
\end{aligned}
$$

It should be noted that approximation of $V_{1}^{(m+1)}=0$ in equation (43) gives the well-known Aitken acceleration. Hence, we have established the relation of the $\varepsilon$-algorithm to the discrete Toda molecule equation (13).

Wynn's result on confluent forms of the $\varepsilon$ - and QD algorithm can also be applied to equation (44). Employing the same dependent variable transformations as in $[26],{ }^{4}$

$$
\begin{aligned}
m & =t / \Delta t \\
S_{m} & =f(t) \\
c_{m+1} & =f(t+\Delta t)-f(t) \\
\varepsilon_{2 n+1}^{(m)} & =\varepsilon_{2 n+1}(t) \\
I_{n}^{(m)} & =\left(I_{n}(t)-1\right) / \Delta t \\
V_{n}^{(m)} & =V_{n}(t) /(\Delta t)^{2}
\end{aligned}
$$

${ }^{3}$ See also Bauer [3] and Cuyt [6].

${ }^{4}$ Variables $n, I_{n}(t)$ and $V_{n}(t)$ correspond to $r, Q_{r}(t)$ and $E_{r}(t)$, respectively, in Wynn's papers. 
and taking the limit $\Delta t \rightarrow 0$, we have a confluent analogue of equation (44),

$$
\varepsilon_{2 n+1}(t)=f(t)+\frac{\dot{f}(t) \mid}{\mid-I_{1}(t)}+\frac{-V_{1}(t) \mid}{\mid-I_{2}(t)}+\frac{-V_{2}(t) \mid}{\mid-I_{3}(t)}+\cdots+\frac{-V_{n-1}(t) \mid}{\mid-I_{n}(t)} .
$$

Now we can explain physically the reason for convergence acceleration from the viewpoint of the Toda molecule equation just as diagonalization of matrices in the QR algorithm. We see from equation (44) that the error between the quantities $\varepsilon_{2 n+1}^{(m)}$ and the limit $S_{\infty}=\lim _{m \rightarrow \infty} S_{m}$ is estimated as

$$
\left|\varepsilon_{2 n+1}^{(m)}-S_{\infty}\right| \cong \prod_{i=1}^{n+1} I_{i}^{(m+1)} \prod_{i=1}^{n} V_{i}^{(m+1)},
$$

or equivalently,

$$
\frac{\left|\varepsilon_{2 n+1}^{(m)}-S_{\infty}\right|}{\left|\varepsilon_{2 n-1}^{(m)}-S_{\infty}\right|} \cong I_{n+1}^{(m+1)} V_{n}^{(m+1)} .
$$

As is mentioned above, each quantity $V_{n}^{(m)}$, which corresponds to

$$
\exp \left(-\left(Q_{n+1}(t)-Q_{n}(t)\right)\right)
$$

in equation (1), decays exponentially to zero as $m \rightarrow \infty$ as does the left hand side of equation (53), which matches exactly the definition of convergence acceleration. Furthermore, we can quantitatively estimate the error $\left|\varepsilon_{2 n+1}^{(m)}-S_{\infty}\right|$. From equations (19), (20) and (44), we find that the estimation is expressed by

$$
\begin{aligned}
& \left|\varepsilon_{2 n+1}^{(m)}-S_{\infty}\right| \\
& =c_{m+1}\left\{\frac{C_{n+1} P_{n+1} \prod_{i=1}^{n}\left(P_{n+1}-P_{i}\right)^{2}}{C_{1} \prod_{i=1}^{n+1}\left(1-P_{i}\right)^{2}}\left(\frac{P_{n+1}}{P_{1}}\right)^{m+1}+O\left(\left(\frac{P_{n+2}}{P_{1}}\right)^{m}\right)\right\} \\
& =\frac{C_{n+1} \prod_{i=1}^{n}\left(P_{n+1}-P_{i}\right)^{2}}{\prod_{i=1}^{n+1}\left(1-P_{i}\right)^{2}} P_{n+1}^{m+2}+O\left(\max \left[P_{n+1} P_{2} / P_{1}, P_{n+2}\right]^{m}\right)
\end{aligned}
$$

Under the assumptions given by equations (18), (22) and (39), we see that inequalities,

$$
-1 \leq P_{1} \leq 1,-1<P_{j}<1(j \geq 2)
$$

hold. It is interesting to note that the case $P_{1}=1$ is exceptional in the sense that the $\varepsilon$-algorithm cannot accelerate convergence of logarithmic sequences.

It is also shown from equation (44) that the discrete Toda molecule equation (38) also serves as a convergence accelerator of a given sequence $S_{m}=\sum_{i=0}^{m} c_{i}$. We 
construct sequences $A_{n}^{(m)}$ and $B_{n}^{(m)}$ according to the following algorithm:

$$
\begin{aligned}
& \text { step 1. } A_{0}^{(m)} \leftarrow S_{m}, B_{0}^{(m)} \leftarrow 1, \\
& A_{1}^{(m)} \leftarrow S_{m+1}, B_{1}^{(m)} \leftarrow 1, \\
& \text { step 2. }\left\{\begin{array}{l}
A_{2 n}^{(m)} \leftarrow A_{2 n-1}^{(m)}-I_{n}^{(m+1)} A_{2 n-2}^{(m)}, \\
B_{2 n}^{(m)} \leftarrow B_{2 n-1}^{(m)}-I_{n}^{(m+1)} B_{2 n-2}^{(m)},
\end{array}\right. \\
& \left\{\begin{array}{lll}
A_{2 n+1}^{(m)} \leftarrow A_{2 n}^{(m)}-V_{n}^{(m+1)} A_{2 n-1}^{(m)}, \\
B_{2 n+1}^{(m)} \leftarrow B_{2 n}^{(m)}-V_{n}^{(m+1)} B_{2 n-1}^{(m)}
\end{array},\right.
\end{aligned}
$$

where the quantities $\left(I_{n}^{(m)}, V_{n}^{(m)}\right)$ are determined by recurrence relations (38). The sequence $\left\{A_{n}^{(m)} / B_{n}^{(m)}\right\}_{n=0,1,2, \ldots}$ converges more rapidly to the limit $S_{\infty}$ as $n \rightarrow$ $\infty$ than the original sequence. Even though this procedure is equivalent to the $\varepsilon$-algorithm (9), its merit is that it does not involve singularities, while the quantities $\varepsilon_{2 n}^{(m)}$ diverge in the $\varepsilon$-algorithm.

The Toda molecule equation, though it may not have attracted attention in a physical sense, stands for a dynamical system which converges to a diagonal matrix and appears in other fields of mathematical engineering. In the future we should study such molecule-type integrable systems, which are expected to find many applications.

\section{BIBLIOGRAPHY}

[1] M. Arai, K. Okamoto and Y. Kametaka, Aitken-Steffenson Acceleration and a New Addition Formula for Fibonacci Numbers, Proc. Japan. Acad. 62 Ser. A (1986) 5-7. MR 87g:11028

[2] G. A. Baker Jr. and P. Graves-Morris, Padé Approximants Part I : Basic Theory, AddisonWesley, Massachusetts, 1981. MR 83a:41009a

[3] F. L. Bauer, The quotient-difference and epsilon algorithms, On Numerical Approximation, R. E. Langer, ed., University of Wisconsin Press, Madison, 1959, 361-370. MR 21:1384

[4] C. Brezinski, Padé-Type Approximation and General Orthogonal Polynomials, BirkhäuserVerlag, Basel, 1980. MR 82a:41017

[5] C. Brezinski and M. Redivo Zaglia, Extrapolation Methods. Theory and Practice, NorthHolland, Amsterdam, 1991. MR 93d:65001

[6] A. Cuyt, The Mechanism of the Multivariate Padé Process, Lecture notes in mathematics Vol. 1071, Padé Approximation and its Applications, H. Werner and H. J. Bünger, ed., Springer, Berlin, 1984, 95-103. MR 85j:41034

[7] H. Flaschka, The Toda lattice. II. Existence of Integrals, Phys. Rev. B, 9 (1974) 1924-1925. MR 53:12412

[8] W. B. Gragg, The Padé table and its relation to certain algorithms of numerical analysis, SIAM Review 14 (1972) 1-62. MR 46:4693

[9] R. Hirota and J. Satsuma, A Variety of Nonlinear Network Equations Generated from the Bäcklund Transformation for the Toda Lattice, Suppl. Prog. Theor. Phys. 59 (1976) 64-100.

[10] R. Hirota, Direct Method in Soliton Theory, Iwanami, Tokyo, 1992 [in Japanese].

[11] R. Hirota, S. Tsujimoto and T. Imai, Difference scheme of soliton equations, RIMS Kokyuroku, 822 (1992) 144-152. MR 95j:58070

[12] Y. Nakamura, A tau-function of the finite nonperiodic Toda lattice, Phys. Lett. A 195 (1994) 346-350. MR 95j:58078

[13] Y. Nakamura, The BCH-Goppa decoding as a moment problem and a tau-function over finite fields, Phys. Lett. A 223 (1996) 75-81. MR 97k:94074

[14] A. Nagai and J. Satsuma, Discrete soliton equations and convergence acceleration algorithms, Phys. Lett. A 209 (1995) 305-312. MR 96h:65006

[15] V. Papageorgiou, B. Grammaticos and A. Ramani, Integrable lattices and convergence acceleration algorithms Phys. Lett. A 179 (1993) 111-115. MR 94b:65009

[16] O. Perron, Die Lehre von den Kettenbrüchen, Taubner, Leipzig, 1929. 
[17] H. Rutishauser, Der Quotienten-Differenzen-Algorithmus, Z.A.M.P. 5 (1954) 233-251. MR 16:176c

[18] D. Shanks, Nonlinear transformations of divergent and slowly convergent sequences, J. Math. Phys. 34 (1955) 1-42. MR 16:961e

[19] K. Sogo, Toda Molecule Equation and Quotient-Difference Method, J. Phys. Soc. Jpn. 62 (1993) 1081-1084. MR 94i:82022

[20] W. W. Symes, The $Q R$ algorithm and scattering for the finite nonperiodic Toda lattice, Physica 4D (1982) 275-280. MR 83h:58053

[21] M. Toda, Waves in Nonlinear Lattice, Prog. Thoer. Phys. Suppl. 45 (1970) 174-200.

[22] H. Togawa, Matrix Computations, Ohm, Tokyo, 1971 [in Japanese].

[23] H. S. Wall, Analytic Theory of Continued Fractions, Chelsea, Bronx, N. Y. 1948. MR 10:32d

[24] D. S. Watkins and L. Elsner, On Rutishauser's Approach to Self-Similar Flows, SIAM J. Matrix Anal. Appl. 11 (1990) 301-311. MR 91e:58145

[25] P. Wynn, On a device for computing the $e_{m}\left(S_{n}\right)$ transformations, Math. Tables Aids Comput. 10 (1956) 91-96. MR 18:801e

[26] P. Wynn, Confluent Forms of Certain Non-linear Algorithms, Arch. Math. 11 (1960) 223236. MR 23:B1113

[27] P. Wynn, A Note on a Confluent Form of the $\varepsilon$-algorithm, Arch. Math. 11 (1960) 237-240. MR 23:B1114

[28] P. Wynn, Upon a Second Confluent Form of the $\varepsilon$-algorithm, Proc. Glasgow Math. Soc. 5 (1962) 160-165. MR 25:2689

[29] P. Wynn, On a Connection between the First and Second Confluent Forms of the $\varepsilon$-algorithm, Nieuw Archief voor Wiskunde 11 (1963) 19-21. MR 26:6641

[30] P. Wynn, Partial Differential Equations Associated with Certain Non-Linear Algorithms, Z. Angew. Math. Phys. 15(1964) 273-289. MR 29:4217

Department of Mathematical Sciences, University of Tokyo, Komaba 3-8-1, MeguroKU, TOKYO 153, JAPAN

E-mail address: slime@poisson.ms.u-tokyo.ac.jp

E-mail address: toki@sunflower.t.u-tokyo.ac.jp

E-mail address: satsuma@poisson.ms.u-tokyo.ac.jp 